\title{
A Case of Bilateral Postauricular Sinuses
}

\author{
Hong Jun Kim¹, Jae Heon Lee ${ }^{2}$, Hyun Sang $\mathrm{Cho}^{2}$ and In Seok Moon ${ }^{1}$ \\ ${ }^{1}$ Department of Otorhinolaryngology, Yonsei University College of Medicine, Seoul, \\ ${ }^{2}$ Department of Otorhinolaryngology-Head and Neck Surgery, Chung-Ang University College of Medicine, Seoul, Korea
}

\author{
Received July 9,2012 \\ Revised August 27, 2012 \\ Accepted September 2, 2012
}

Preauricular sinuses are common congenital malformations that usually occur at the anterior margin of the ascending limb of the helix. There are rare cases in which the location of the preauricular sinus is posterior to the tragus, and the direction of the sinus tract is toward the posterior. This variant type of preauricular sinus is called, 'postauricular sinus'. Postauricular sinus is uncommon. Thus, diagnosis and treatment may be delayed due to its location of its opening and atypical clinical symptoms. Typical treatment is a single stage operation through bidirectional skin incision. However, we found out that according to the length and invasion depth of the sinus tract, there was an option between unidirectional and bidirectional skin incisions that we may choose from.

Korean J Audiol 2012;16:99-101

Tel $+82-2-2228-3627$

Fax +82-2-393-0580

KEY WORDS: Preauricular sinus · Variant · Excision.

\section{Introduction}

Preauricular sinus is a congenital disorder, first reported by Heusinger ${ }^{1)}$ in 1864 , and known to be occurred by an abnormal union of the hillock of his in the prenatal auricle formation process. The inner surface of the preauricular sinus is composed of squamous epithelium, and the opening of the sinus is usually located in the anterior to the crus of helix and anterior-superior to the tragus. It may occur unilaterally or bilaterally. ${ }^{2)}$ Usually, it is asymptomatic, and specific treatment is not needed if it is not infected. However, when the sinus becomes infected, it may cause pain that is associated with swelling, and because it reoccurs often, surgical treatment should be considered. $^{2)}$

There is a variant of the preauricular sinus called 'postauricular sinus', and unlike the preauricular sinus, the sinus opening develops posterior to the tragus and near the crus of helix. Also, the direction of this variant is from the opening toward the retroauricular area. ${ }^{3)}$ Unlike the typical symptoms of preauricular sinus, this variant type sinus occurs as a swelling and erythema of retroauricular area. Therefore, differential diagnosis is necessary for lymphadenitis, epidermoid cyst, steatocystoma, and brachial cleft cyst, which may present as a mass or cyst in the retroauricular area. In rare cases, if there is recurrence, even after adequate antibiotics treatment, incision and drainage, and excision is performed for mass like lesions in the retroauricular area; postauricular sinus should be considered as the cause.

We report a case with review of the literature of a patient with bilaterally developed variant type preauricular sinuses, which were completely removed without recurrence.

\section{Case Report}

A 27 year old male visited the department of Otorhinolaryngology-Head and Neck Surgery, Chung-Ang University hospital, with complaints of painful swelling, erythema, and tenderness on the left retroauricular area. He had a history of incision and drainage twice in the right ear, and once in the left ear due to similar symptoms in the past. On physical examination, there was swelling and tenderness in the left retroauricular area. Further, in the center of the crus of helix, there was a small opening showing pus drainage. There was no swelling or tenderness on the right auricle, but there was a small opening in the center of the crus of helix.

Since there were sinus openings in both crus of helix (Fig. 1), manifestations of retroauricular area swelling, and history of recurrent infection, the patient was prediagnosed as the bilateral variant type of preauricular sinus, called 'postauricular sinus'. Surgery was performed after one week of antibiotics 

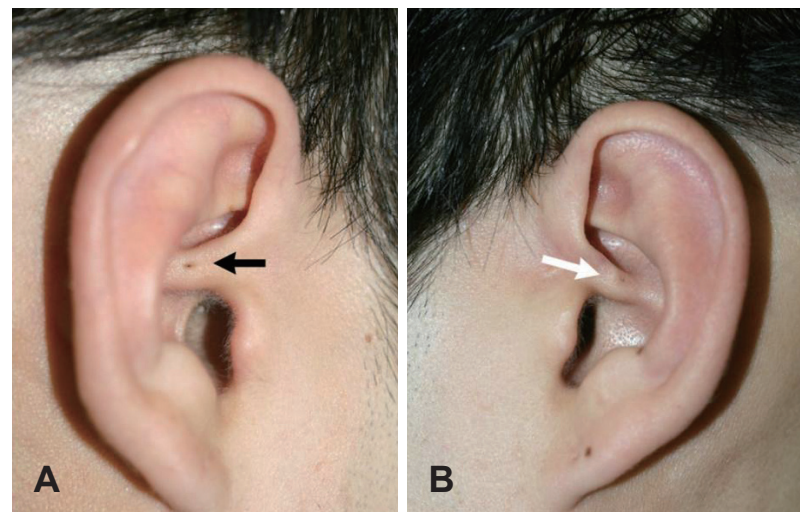

Fig. 1. Gross photograph of the patient shows $(A)$ auricular pit on right middle area on the crus of helix (black arrow) and (B) auricular pit on left middle area on the crus of helix (white arrow).
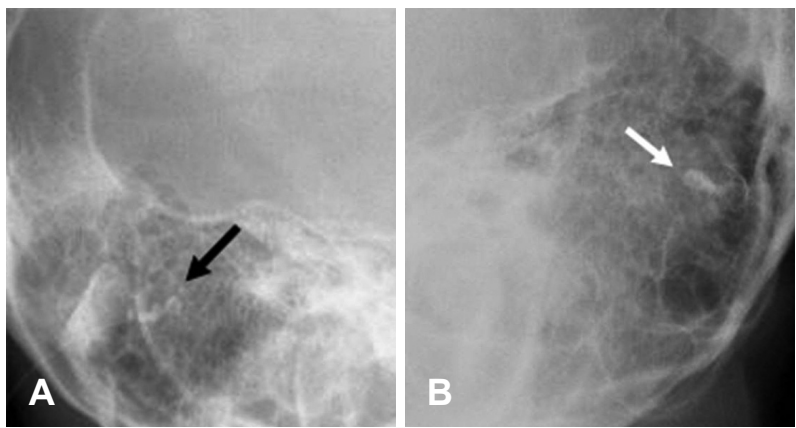

Fig. 2. Preoperative fistulography shows $1 \mathrm{~cm}$ tubular shape fistula (black arrow) in right upper portion of EAC (A) and $0.5 \mathrm{~cm}$ tubular shape fistula (white arrow) in left upper portion of EAC (B). EAC: external auditory canal.

treatment and acute inflammation was relieved. Fistulography was performed in order to identify the location of the sinus and the length of the sinus tract, and there were a $0.5 \mathrm{~cm}, 1.0 \mathrm{~cm}$ sized sinus tract for each right and left side, heading towards the posterior-middle portion of the auricles (Fig. 2).

During the surgery, local anesthesia was performed around the left sinus opening and retroauricular area. Then, gentian violet was injected into the sinus tract for dyeing. Perpendicular-oval shaped incision, including the opening of the sinus, was done and excision was preceded by identifying the dyed sinus. The direction of the sinus tract could be indentified using a lacrimal probe. The sinus was observed to penetrate the cartilage of the crus of helix and directed toward posterior part of the auricle as in the sinus form. Retroauricular incision was performed for complete removal of the sinus, and cartilage of the crus of helix was also removed (Fig. 3). Then, a drain was inserted and primary closure was performed. Same procedure was performed for the right side, but upon the insertion of the probe, the length of the sinus was small, and it was observed that it invaded to invade only a portion of cartilage of the crus of helix. Therefore, only an incision around the sinus opening was used completely to remove the sinus (Fig. 3). The patient

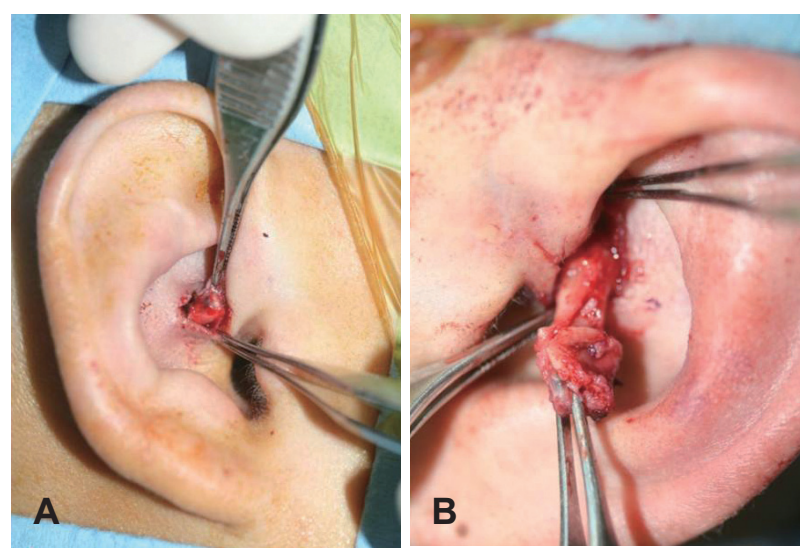

Fig. 3. During surgery, (A) shows right fistular tract being dissected only by preauricular approach. (B) Shows left fistular tract being dissected via dual approach through postauricular and preauricular incisions.
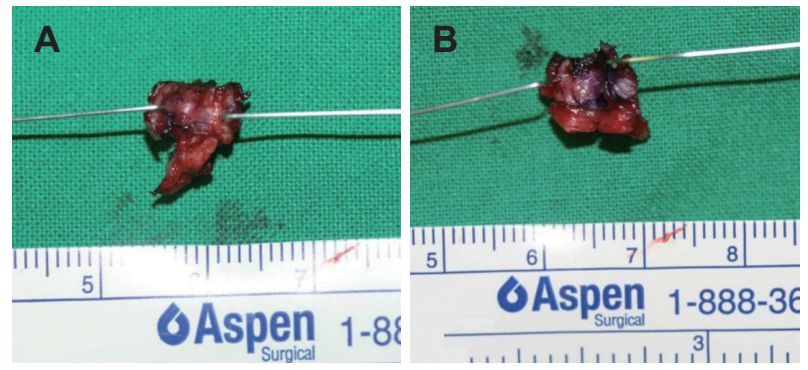

Fig. 4. Gross findings shows $(A)$ right fistula measuring $0.9 \times 1.2 \times$ $0.8 \mathrm{~cm}$ (B) left fistula measuring $1.2 \times 1.3 \times 0.8 \mathrm{~cm}$.

was discharged without complications and total stitch off was done 7 days after the surgery. Histopathologic studies diagnosis was confirmed it as preauricular sinus, since the sinus tract lined with chronic inflammatory squamous epithelium (Fig. 4, 5), and 12 months after the surgery, the patient is on follow up without complications and signs of recurrence.

\section{Discussion}

Preauricular sinus is known to form due to the incomplete fusion of hillock of his, which originated from the first and second branchial arch. ${ }^{1,2)}$

Frequency of preauricular sinus differs depending on race, $0.1-9 \%$ in the US, $0.9 \%$ in the UK, and $4-10 \%$ in Asia and parts of Africa. ${ }^{2,4)}$ Frequency is known to be higher in black people and Asians compared to white people. In Korea, frequency is about $5 \%$ and has been commonly reported.

In a variant of preauricular sinus called 'postauricular sinus', the entrance of the sinus is located posterior to the helix and tragus, but its pathogenesis is known to be similar to the typical preauricular sinus. However, its frequency is known to be very rare, compared to preauricular sinus. ${ }^{3)}$ In abroad, Chang and $\mathrm{Wu}^{6}$ have reported 3 cases of preauricular sinus in which 


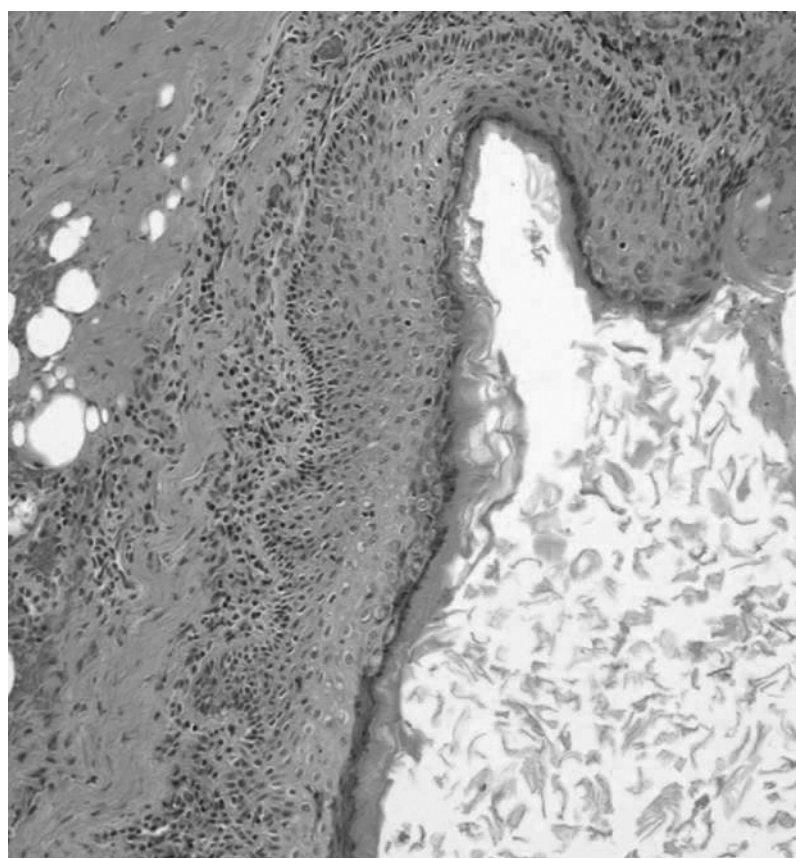

Fig. 5. Pathologic finding shows preauricular sinus lined squamous epithelium with hyperkeratosis and parakeratosis (hematoxylin-eosin; magnification $200 \times$ ).

the sinus opening was located in the crus of helix and the sinus was placed posterior to the auricle. In Korea, Yeo, et al. ${ }^{7)}$ have reported 8 cases with sinus opening in the auricle, out of the 206 preauricular sinus patients.

The reasons for the difference in the frequency for preauricular sinus and postauricular sinus are the following; first, postauricular sinus is not separately classified with typical preauricular sinus. Second, in postauricular sinus, the location of the sinus is not consistent, compared to typical preauricular sinus and classification criteria do not exist. And third, misdiagnosis is made for other disease with cystic lesions in the retroauricular area.

Recently, Choi, et al. ${ }^{3)}$ have named a perpendicular line posterior to the tragus and ascending limb of the helix as extended tragal line. It was classified as preauricular sinus when the opening of the fistula was located anterior to the extended tragal line and classified as postauricular sinus when the opening was posterior to this line. Since the sinus opening is different for postauricular and preauricular sinus, the location of sinus tract is different, as well. In postauricular sinus, the direction of the sinus is toward the posterior-center portion or posterior-inferior portion of the auricle from the crus of helix. Therefore, fistulography prior to surgery may be helpful to know such variable route and length of the sinus.
It commonly forms branches, and recurrence is not uncommon due to remnant branches after the surgery. ${ }^{8}$ Principle of the treatment in preauricular sinus is to remove the whole sinus, including its branches. ${ }^{2)}$ In postauricular sinus, complete excision is more difficult due to its variable route and sinus is surrounded by more compact tissues. Methylene blue, probe, and surgical microscope make it easier to find the tract and to remove the sinus completely. ${ }^{8,9)}$ In postauricular sinus, the sinus opening is located in the center of the crus of helix, or its surrounding; so it has a depressed shape, and the sinus tract is either attached to the cartilage or penetrated. Therefore, the portion of auricle cartilage needs to be removed, and removal via anterior-posterior approach of the auricle is necessary to completely remove the postauricular sinus. ${ }^{3)}$

Generally, it is known that bidirectional approach is necessary to remove the postauricular sinus, but we decided to perform the removal via unidirectional approach for the right ear, since the sinus tract was not in great length according to fistulography. For the left ear, since the sinus tract was directed toward the posterior, which included the cartilage and had a big cystic lesion, removal via bidirectional approach was inevitable.

Unlike preauricular sinus, the tract of the postauricular sinus has different locations and directions. As such, careful observation is needed and selective surgical procedure is necessary since the removal via unilateral skin incision is possible according to the length and invasiveness of the sinus.

\section{REFERENCES}

1) Heusinger HK. Fisteln von Noch Nicht Beobachteter Form. Virchows Arch 1864;29:358.

2) Scheinfeld NS, Silverberg NB, Weinberg JM, Nozad V. The preauricular sinus: a review of its clinical presentation, treatment, and associations. Pediatr Dermatol 2004;21:191-6.

3) Choi SJ, Choung YH, Park K, Bae J, Park HY. The variant type of preauricular sinus: postauricular sinus. Laryngoscope 2007;117: 1798-802.

4) Ewing MR. Congenital sinuses of the external ear. J Laryngol Otol 1946;61:18-23.

5) Yang EY. An epidemiologic survey of congenital preauricular fistula. Korean J Otolaryngol 1977;20:1-4.

6) Chang $\mathrm{PH}, \mathrm{Wu} \mathrm{CM}$. An insidious preauricular sinus presenting as an infected postauricular cyst. Int J Clin Pract 2005;59:370-2.

7) Yeo SW, Jun BC, Park SN, Lee JH, Song CE, Chang KH, et al. The preauricular sinus: factors contributing to recurrence after surgery. Am J Otolaryngol 2006;27:396-400.

8) Cho YB, Baik J, Cho Y, Lim HJ, Na AS. Clinical study on surgery of infected congenital preauricular fistula. Korean J Audial 1998;2:17780.

9) Prasad S, Grundfast K, Milmoe G. Management of congenital preauricular pit and sinus tract in children. Laryngoscope 1990;100:320-1. 